\title{
Osteoporosis-related life habits and knowledge about osteoporosis among women in Tabriz, Iran
}

Rafraf $M^{a}$, Bazyun $B^{b}$ and Afsharnia $F^{c}$

a Nutritional Research Center, Tabriz University of Medical Sciences, Iran

${ }^{b}$ Faculty of Health and Nutrition, Tabriz University of Medical Sciences, Iran

c Public health centre, Tabriz University of Medical Sciences, Tabriz, Iran

\begin{abstract}
Introduction: The objectives of this study were to investigate the calcium intake, physical activity level and knowledge about osteoporosis in women who attended health centers in Tabriz, Iran. Materials and Methods: Three hundred ninety-nine women of childbearing age were studied. Information was collected through face

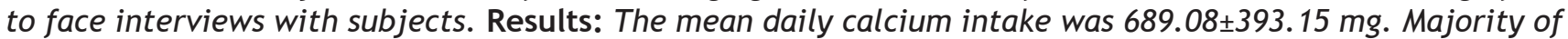
subjects had low or moderate physical activity levels. Only $8 \%$ of women reported high physical activity level. Knowledge about osteoporosis in majority of subjects (63.2\%) was at moderate level. Educational status of subjects was positively correlated with calcium intake. There were no significant statistics relationship between calcium intake and physical activity with other studied variables. Conclusion: Public health programs are suggested to improve calcium intake of women and elevating healthy life styles. Establishment of educational workshops to health care providers and encourage and obligate them to proper consultation with women is suggested as a suitable approach for prevention of osteoporosis.
\end{abstract}

KEYWORDS: Osteoporosis, Calcium intake, Physical activity, Knowledge, women

\section{INTRODUCTION}

Osteoporosis is a systemic metabolic disease resulting in low bone mass, and deterioration of bone structure, which increases the risk for fracture..$^{1-4}$ It is more prevalent among women than among men. ${ }^{3}$ Some risk factors for osteoporosis include female sex, Caucasian or Asiatic race, advancing age, family history of osteoporosis or fragility fractures, sedentary life style, menopause before age 45 years, prolonged lactation, diet low in calcium and vitamin D. ${ }^{3,5}$

Worldwide variation in the incidence and prevalence of osteoporosis is difficult to determine because of problems with definition and diagnosis. The most useful way of comparing osteoporosis prevalence between populations is to use fracture rates in older people. However, because osteoporosis is usually not life-threatening, quantitative data from developing countries are scarce. The prevalence of osteoporosis in less developed and developing countries is not clear, as there are few studies in these populations. However, ethnic differences in BMD are well known. Blacks have greater BMD than Caucasians. Hispanics are similar to Caucasians, while Asians have the lowest BMD. ${ }^{5}$

Corresponding author;

Dr Rafraf Maryam,

Faculty of Health and Nutrition,

Tabriz University of Medical Sciences,

Tabriz, Islamic Republic of Iran.

E-mail address: rafrafm@tbzmed.ac.ir
The real incidence of osteoporotic fractures in Iran might be underestimated because of poor health records in our country. However, The results of one study on 553 subjects (34\% men, 66\% women) aged 20-69 years, randomly selected from 50 blocks in Tehran (the capital of Iran), indicated that the prevalence of osteoporosis of the lumbar spine and femur was $32.4 \%$ and $5.9 \%$ in women and $9.4 \%$ and $3.1 \%$ in men respectively. ${ }^{6}$

The first step in the prevention of osteoporosis in women should be making them aware of the risk factors. ${ }^{7,8}$ There is evidence suggesting that osteoporosis knowledge is one contributor to osteoporosis preventive behavior, though this is not a clear-cut relationship. ${ }^{9}$ Some studies on women of different countries indicated that knowledge about osteoporosis risk factors is limited, irrespective of age. ${ }^{10-16}$ Healthy life styles in child bearing age women may protect them against osteoporosis in later life. Bearing in mind the lack of reliable epidemiological data about this disease in our country, the present study aims to investigate osteoporosis-related life habits (including calcium intake and exercise) and knowledge about osteoporosis among women of childbearing age in Tabriz, the capital of Azerbaijan province in the northwest of Iran.

\section{MATERIALS AND METHODS}

This cross-sectional study was performed on 399 healthy non-lactating, non-pregnant childbearing age women aged 15-49 years. The ethical committee of Tabriz University of Medical Sciences approved the study protocol. Fifteen health centers (30\% of total 
health centers) were randomly selected and healthy childbearing age women who attended these health centers during 3 months of study were included. Informed oral consent was obtained from all subjects. Information about general characteristics of women was gathered by interviewing the subjects and physical activity level was obtained by international physical activity questionnaire: IPAQ. ${ }^{17}$ Daily calcium intake was assessed by 24-hour recall method. ${ }^{18}$ Knowledge about osteoporosis prevention and risk factors was assessed by questionnaire which consisted of 21 items. The questions were set according to previous studies on osteoporosis. $.^{3},{ }^{10}$ Each question had one correct answer. The total score of the questionnaire was 21; equal to $100 \%$. The score under $50 \%$, between $50-75 \%$, and $>75$ were classified as low, moderate and high knowledge level; respectively.

Data were analyzed with SPSS for windows version 11.5. The FP II (food processor II) software was used for analyzing diet questionnaires. Mean and frequency of variables were determined. Pearson correlation coefficient and Spearman correlation coefficient used for assessing correlation between variables having normal and non-normal distribution, respectively. Results considered significant, if $p<0.05$.

\section{RESULTS}

The participant characteristics are shown in Table I. The mean age of studied women was 33.13 years. Majority of subjects had educational level at grade 8 or less. Calcium and vitamin D supplements were consumed by $11.8 \%$ and $6.3 \%$ of subjects, respectively.

Table I. Characteristics of participants $(n=399)$

$\begin{array}{ll}\text { Characteristics } & \text { Mean (SD) } \\ & \text { or } \%\end{array}$

Age

33.13

Education level \%

Illiterate

9.3

Grade 5 or less

24.6

Grade 8

22.8

Completed grade 12

35.8

University or other tertiary institution 7.5

Calcium supplement users $\%$

11.8

Vitamin D supplement users \%

6.3

Table II summarizes distribution of subjects based on daily calcium intake, weekly physical activity, and knowledge level. Most subjects (50.7\%) had low calcium intake [less than $600 \mathrm{mg} /$ day which is $60 \%$ of the recommended daily intake (DRI), $1000 \mathrm{mg} / \mathrm{day}] .{ }^{19} \mathrm{Lev}$ el of physical activity was moderate or low in majority of women, $(42.6 \%$, and $37.8 \%$, respectively), and $19.6 \%$ had physical activity at high level. The mean of total scores of knowledge regarding osteoporosis were $12.14 \pm 2.98$. Most interviewed subjects $(63.2 \%)$ had moderate knowledge about osteoporosis regarding the concept of the disorder and its risk factors, sex-related factors and prevention behaviors.

Table II. Distribution of subjects based on calcium intake, physical activity and knowledge levels

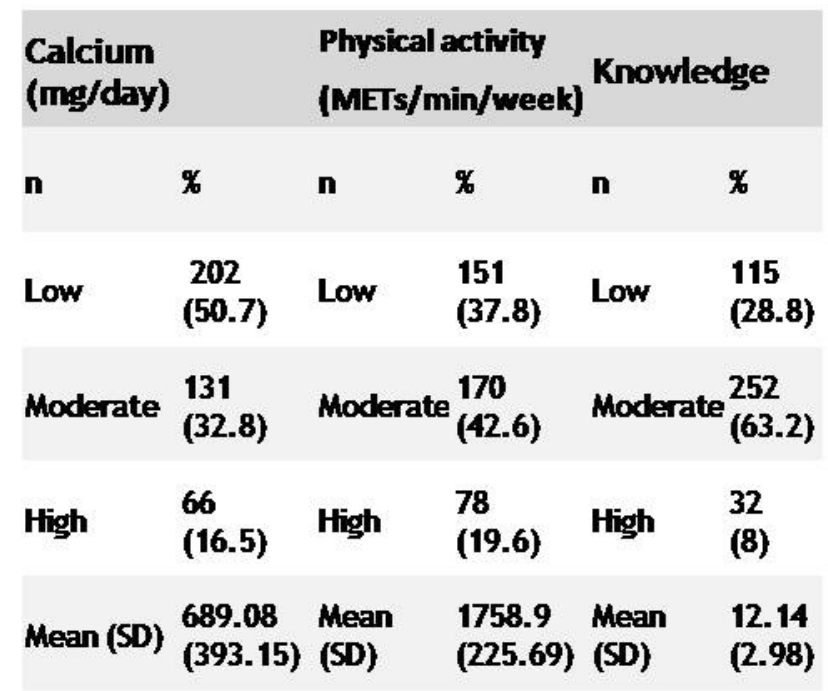

There were positive significant correlation between calcium intake and educational levels of women (Spearman correlation coefficient; $r=0.02, p<0.001$ ). No significant relationship was found between calcium intake and physical activity with other studied variables such as age and knowledge level (data are not shown).

\section{DISCUSSION}

Osteoporosis has recently been recognized as a major public health problem by some governments and health care providers. ${ }^{20}$ Prevention is the most costeffective means of managing osteoporosis. ${ }^{7}$ Two important preventive habits are diet or supplements containing adequate levels of calcium and vitamin $D$, and weight-bearing exercise (e.g. going up and down stairs, jogging, aerobic, and isometrics; at least 30 minutes daily). ${ }^{13,15,21-23}$

Our findings (Table II) indicate that, most women interviewed $(50.4 \%)$ had a daily calcium intake of less than $60 \%$ of the recommended level, and calcium or vitamin $D$ supplements were not consumed by majority of subjects, thus increasing the risk of osteoporosis. Studies on women of other countries such as Caucasian and African-American and Hispanic women in the US, and Asian and Caucasian women in Australia, have shown that most of these women did not fulfill the suggested calcium intake.11,12,24 Similarly, non adequate calcium intakes in women have been reported in other studies. $3,{ }^{12,24-28}$ The finding of positive significant correlation between calcium intake and educational levels of women indicates that higher educational level may be associated with healthier diet. Higher educational level may be related to high- 
er income, and therefore choosing better sources of calcium such as milk and milk products. Thus, women with low educational level are at risk of osteoporosis more than others, and so more attention should be focused on elevating women's literacy in our country.

Optimal physical activities are necessary for increasing bone mass and thus perhaps reducing the risk of osteoporosis. According to our findings, majority of subjects had physical level at moderate or low levels. Other studies also reported that few women performed sufficient high-intensity weight bearing physical activity to improve bone mass. $3,7,25,27$

Based on our results (Table II), many women had moderate knowledge about osteoporosis risk factors and preventive behaviors. This knowledge often does not translate to appropriate changes in healthy life habits as it is shown through no significant association between total knowledge scores and calcium intake or physical activity level. Low or moderate levels of knowledge about this disease in women of other countries are also reported by some studies. ${ }^{3,15,29}$ It seems that osteoporosis knowledge is not well internalized among studied women.

As a conclusion, most studied childbearing age women do not achieve the DRI for calcium. Physical activity and knowledge levels about osteoporosis are not sufficient in studied women. Public health strategies should be aimed at improving the calcium intake of women in this age group, and to make appreciated lifestyle changes such as elevating weight bearing physical activity. Educational programs are suggested to encourage primary health care providers to consult with women more effectively about osteoporosis preventions.

\section{Acknowledgements}

We thank the Nutritional Research Center of Tabriz University of Medical Sciences, Tabriz, Islamic Republic of Iran, for financial support, and the women who participated in the study.

\section{REFERENCES}

1. Bonura F. Prevention, screening, and management of osteoporosis: an overview of the current strategies. Postgrad Med J 2009; 121:5-17

2. Gronholz MJ. Prevention, diagnosis, and management of osteoporosis-related fracture: a multifactoral osteopathic approach. J Am Osteopath Assoc 2009; 109:191-3

3. Hernandez-Rauda R, Martinez-Garcia S. Osteoporosis-related life habits and knowledge about osteoporosis among women in EL Salvador: a cross-sectional study. BMC Musculoskelet Disord 2004; 5:29-46

4. W.H.O. Prevention and management of osteoporosis. World Health Organ Tech Rep Ser. 2003; 221: 1-164
5. Handa R, Ali Kalla A, Maalouf G. Osteoporosis in developing countries. Best Pract Res Clin Rheumatol. 2008; 22:693-708

6. Larijani B, Hossein-Nezhad A, Mojtahedi A, et al. Normative data of bone mineral density in healthy population of Tehran, Iran: a cross sectional study. BMC Musculoskelet Disord 2005; 6:38

7. Orces $\mathrm{CH}$, Casas $\mathrm{C}$, Lee $\mathrm{S}$, et al. Determinants of osteoporosis prevention in low-income MexicanAmerican women. South Med J 2003; 96:458-64

8. Covello VT, Peters RG. Women's perceptions of the risks of age-related diseases, including breast cancer: reports from a 3-year research study. Health Commun 2002; 14:377-95

9. Winzenberg TM, Oldenburg B, Frendin S, Jones G. The design of a valid and reliable questionnaire to measure osteoporosis knowledge in women: the osteoporosis knowledge assessment tool (OKAT). BMC Musculoskelet Disord 2003; 4:1-7

10. Terrio K, Auld GW. Osteoporosis knowledge, calcium intake, and weight-bearing physical activity in three age groups of women. J Community Health 2002; 27:307-20

11. Kasper MJ, Peterson MG, Allegrante JP. The need for comprehensive educational osteoporosis preention programs for young women: results from a second osteoporosis prevention survey. Arthritis Rheum 2001; 45:28-34

12. Liew YL, Mann D, Piterman L. Osteoporosis risks; A comparative study of Asian Australian and Caucasian Australian women. Aust Fam Physician.2002; 31:291-3

13. Chang SF, Hong CM, Yang RS. Cross-sectional survey of women in Taiwan with first-degree relatives with osteoporosis: knowledge, health beliefs, and preventive behaviors. J Nurs Res 2007; 15:224-32

14. Cline RR, Worley MM. Osteoporosis health beliefs and self-care behaviors: an exploratory investigation. J Am Pharm Assoc 2006; 46:356-63

15. Von Hurst PR, Wham CA. Attitudes and knowledge about osteoporosis risk prevention: a survey of New Zealand women. Public Health Nutr 2007;10:747-53

16. Chang SF. Knowledge, health beliefs and healthrelated behaviours of first-degree relatives of women suffering from osteoporosis in Taiwan: a questionnaire survey. J Clin Nurs 2008; 17:1280-6

17. International physical activity questionnaire IPAQ: short last 7 days format for use with young and middle -aged adults [online].Accessed at www.ipaq.ki.se

18. Hammond K. Assessment: Dietary and Clinical Data. In: Krause's Food and Nutrition Therapy. Mahan LK, Escott-Stump S. 12th ed. Canada: Saunders, 2008.

19. Gallagber ML. The nutrients and their metabolism. In: Krause's Food and Nutrition Therapy. Mahan LK, Escott-Stump S. 12th ed. Canada: Saunders, 2008.

20. Kutsal YG, Atalay A, Arsalan S, et al. Awareness of osteoporotic patients. Osteoporos Int 2005; 16:128-33 
21. Suleiman S, Nelson M, Li F, Buxton M, Moniz C. Effect of calcium intake and physical activity level on bone mass and turnover in healthy, white, postmenopausal women. Am J Clin Nutr 1997; 66:937-43

22. Carlsson L, Johnson CS. Osteoporosis health beliefs and practices among Korean immigrants in Nova Scotia. J Immigr Health 2004; 6:93-100

23. Sato Y, Tamaki J, Kitayama F, et al. Development of a food-frequency questionnaire to measure the dietary calcium intake of adult Japanese women. Tohoko J Exp Med 2005; 207:217-22

24. Geller SE, Derman R. Knowledge, beliefs, and risk factors for osteoporosis among AfricanAmerican and Hispanic women. J Natl Med Assoc 2001; 93:13-21

25. Saw SM, Hong CY, Lee J, et al. Awareness and health beliefs of women towards osteoporosis. Osteoporos Int 2003; 14:595-601

26. Winzenberg TM, Riley M, Frendin S, et al. Sociodemographic factors associated with calcium intake in premenopausal women: cross-sectional study. Eur J Clin Nutr 2005; 59:463-6

27. Winzenberg TM, Riley M, Frendin S, et al. Sociodemographic factors associated with calcium intake in premenopausal women: crosssectional study. Asia Pac J Clin Nutr 2003;12 suppl:S25

28. Kasper MG, Peterson MG, Allegrante JP, et al. Knowledge, beliefs and behaviors among college women concerning the prevention of osteoporosis. Arch Fam Med 1994; 3:696-702

29. Ungan M, Tumer M. Turkish women's knowledge of osteoporosis. Fam Pract 2001; 18:199-203 